\title{
Sleep Health and Sexual Risk Taking
}

\section{Rossmeisl A and Berg-Cross L*}

Professor of Psychology, Howard University, USA

*Corresponding author: Linda Berg-Cross, PhD, Professor of Psychology, Howard

University, USA, Tel: 301-801-6738; E-mail: lindabergcross@gmail.com

\section{Research Article}

Volume 3 Issue 2

Received Date: February 01, 2018

Published Date: February 22, 2018

\section{Abstract}

The current study explored the relationship between sleep health and sexual risk taking. The study consisted of 104 participants (41 males, 63 females) ages 18-35, who were not presently in a monogamous relationship. Participants completed a series of measures assessing sleep, sexual risk taking, sexual activity, and personality. Results revealed significantly higher levels of sexual risk taking in Unhealthy Sleepers (poor sleep quality and daytime sleepiness) than in Healthy Sleepers (good sleep quality and no daytime sleepiness) for both males and females. However, this relationship was significantly stronger among the women (accounting for $22 \%$ of the variance in risk taking) than among the men (accounting for 3.1\% of the variance in risk taking). Sleepers who reported daytime sleepiness or poor sleep quality (but not both) also showed a number of significant differences in sexual risk taking compared to the other two groups. Implications and recommendations for future research are discussed.

Keywords: Wakefulness; Cognitive Deficits; Judgment; Impulsivity; Implications

\section{Introduction}

Sexual risk taking, a prevalent public health problem throughout the world, has been associated not only with health risks but with depression, low self-esteem and substance abuse [1]. Sleep disorders, on the other hand, are one of the most common reasons people seek medical attention [2]. Poor sleep can result in negative moods, cognitive deficits, deviant behaviors, health problems and psychological abuse [3-7]. While the deleterious effects of poor sleep health and risky sexual behaviors have been extensively explored independently of one another, there is a marked lack of research considering the two variables together. It is important to consider if poor sleep plays a role in one's decision to engage in sexual risk taking.

While direct research in this area is lacking, there is research attesting to the strong relationship between poor sleep, poor cognitive functioning, stress and impulsiveness as well as research documenting the relationship between engaging in risky sex, poor cognitive functioning, stress and impulsiveness. A meta-analysis of 176 articles that explored the effects of sleep deprivation on cognitive functioning confirmed that the cognitive domain of simple, sustained attention exhibited the greatest impairment following short-term sleep deprivation [4]. There were significant effects of sleep deprivation on other cognitive domains, as well, including complex attention, processing speed, working memory, and short-term memory.

Killgore et al. (2006) studied 48 healthy men and women ages 19-39, and found sleep deprivation had a significant impact on decision-making abilities [8]. Prior to undergoing sleep deprivation, participants completed the Iowa Gambling Task (IGT) after 8 hours of sleep. 


\section{Psychology \& Psychological Research International Journal}

Participants were then administered a IGT every other hour during a period continuous wakefulness. The IGT is composed of four decks of cards, two of which are high pay-off, high-risk (disadvantageous), and two that are low pay-off, low-risk (advantageous). After 49 plus hours of continuous wakefulness, compared to their baseline scores, individuals' performance on the IGT significantly declined following sleep deprivation, as they shifted from the low-payoff, low-risk decks to selecting cards from the high-payoff, high-risk decks. After sleep deprivation, participants exhibited marked impairments in their ability to assess the benefits of short-term gains versus the costs of long-term losses, which are consequences consistent with deficits in judgment, risk perception and appraisal, inhibition, and decision-making processes. Thus, sleep deprivation does appear to impair rational decision-making abilities.

Note that within the sleep research field, there are three main independent variables that are studied and manipulated: sleep deprivation, which measures the total number of hours slept at one stretch or the total number of hours awake without sleep; sleep quality, which assesses sleep architecture and sleep disorders such as sleep apnea, restless leg, narcolepsy, insomnia, etc.; and daytime sleepiness, which assesses how alert and refreshed a person feels during the day. Both daytime sleepiness and sleep quality are most often associated with sleep deprivation. The current study is the first of its kind to examine how behavior is impacted by what we are terming, sleep health; the combination of sleep quality and daytime sleepiness. We examine four sleep health conditions (Healthy Sleepers, Unhealthy Sleepers, Impaired Sleepers, and Drowsy sleepers), to more closely replicate actual sleep ecologies and assess the impact of multiple sleep features on behavior.

The predictors of risky sexual behavior show great overlap with the consequences of unhealthy sleep patterns. It appears that unhealthy sleep sets one up to be vulnerable to the stimuli controlling decisions on risky sexual practices. Studies have shown that stress reduction and affect management are among the top reasons people state for engaging in sexual activity (risky or unrisky) [911]. It seems reasonable to hypothesize that individuals who endorse these sexual motives are likely to report higher levels of sexual activity following sleep deprivation, as lack of sleep primes the stress response and decreases positive affect.
Typical of the studies examining the predictors of risky sexual behavior is the work of Macapagal, et al. (2010), who investigated how decision making to sexually arousing stimuli is affected by varying levels of impulsivity and cognitive ability [12]. Participants were assessed for impulsivity and cognitive ability and then placed in one of four conditions: neutral film followed by a neutral learning task, neutral film and sexual learning task, sexual film and neutral learning task, or a sexual film and sexual learning task. The findings revealed that when presented with a sexually arousing stimulus in the sexual learning task condition, individuals high in impulsivity, low in abstract intellectual ability, and high in sexual excitation, achieved a significantly lower performance on the learning tasks. Extrapolating the results of such studies to the real world, it appears that the combination of increased impulsivity and decreased problem-solving abilities would greatly increase the likelihood of risky sexual activity. The current study explores how the negative impact of sleep problems increases the probability of risky sexual activity.

\section{Hypotheses}

Risky sexual behavior is likely to have direct links to sleep health since social judgment, risk perception, and inhibition skills are compromised by poor sleep health. Thus, it is hypothesized that sleep heath will be positively correlated with sexual risk taking.

\section{Participants}

Participants in this study consisted of 104 males $(39.4 \%, N=41)$ and females $(60.6 \%, N=63)$ ages $18-35$. Participants were assigned to one of four sleep groups according to their respective PSQI and ESS scores: healthy sleepers $(N=10)$, PSQI $<5$, ESS $<10$, unhealthy sleepers $(N=44)$, PSQI $\geq 5$, ESS $\geq 10$, drowsy sleepers $(N=8)$, PSQI $<5$, ESS $\geq 10$, and qualitatively impaired sleepers $(N=$ 42 ), PSQI $\geq 5$, ESS $<10$. The healthy sleepers group is comprised of individuals with "good sleep quality" and "low daytime sleepiness," while the unhealthy sleepers group contains participants with "poor sleep quality" and "high daytime sleepiness." The drowsy sleepers group contains individuals with "good sleep quality" and "high daytime sleepiness," and the qualitatively impaired group included participants with "poor sleep quality" and "low daytime sleepiness." The creation of these groups allowed us to examine the different components of sleep health with respect to sexual risk taking. 


\section{Psychology \& Psychological Research International Journal}

\section{Procedure}

A description of the study with a link to the survey was posted on various social networking sites, such as Facebook and Twitter, and was subsequently reposted by other users. The study was completed online at SurveyMonkey.com, at the participant's convenience. Before beginning the study, participants were presented the informed consent form, which they signed and submitted electronically. The measures were presented in randomized sequence to control for potential order effects.

\section{Measures}

\section{Demographics Sheet}

This brief 16-item questionnaire was used to collect relevant personal information regarding basic participant characteristics, including sex, age, ethnicity, religious affiliation, education, income, sexual orientation, and sexual history.

\section{Pittsburgh Sleep Quality Index}

The Pittsburgh Sleep Quality Index (PSQI) was developed to assess sleep quality over a 1-month period, as a way to distinguish between good and poor sleepers. The PSQI is a 24-item survey, with 19 self-reported questions and 5 questions answered by an individual with whom the respondent shares a bed or room, and takes about 5-10 minutes to complete, Empirical evidence demonstrating the psychometric properties of the PSQI has established its utility as a reliable, valid, and standardized measure of subjective sleep quality [13]. Reliability of the PSQI was established through good internal consistency of the global PSQI score $(\alpha=0.83)$, correlations between the component scales and global score ( $r$ between 0.35 and 0.76 ) and individual items and global score ( $r$ between 0.20 and 0.66). Test-retest reliability over a period of 28 days was also adequate for the global score $(\alpha=0.85)$ and component scales $(\alpha=$ $0.65-0.84$ ). Convergent validity of the PSQI was shown through significant correlations between the sleep latency component scale score and polysomnographic measures $(r=0.33)$, and the global PSQI score with sleep latency as measured using polysomnography $(r=0.20)$. Additionally, the PSQI demonstrated good diagnostic validity, as the component scales differed significantly among the diagnostic groups, and a global PSQI score greater than 5 accurately identified $84 \%$ of patients struggling with initiating or maintaining sleep, $89 \%$ with excessive sleepiness, and $97 \%$ with depression $[14,15]$.

\section{Epworth Sleepiness Scale}

The Epworth Sleepiness Scale ESS is brief 8-item selfreport, asking individuals to rate on a four point Likert scale how likely they are to doze or fall asleep in eight different real life situations, and takes about 5 minutes to complete. Research has shown ESS scores to differ significantly among controls and patients with various sleep disorders $(p<.000)$, as well as differentiate between different sleep disorders $(p<.05)$. Additionally, significant negative correlations were found between ESS scores and sleep latency at night $(r=-.379, p<.01)$, and ESS scores and sleep latency during the day $(r=-.514, p<.01)$ [16].

\section{Sexual Risk Survey}

The Sexual Risk Survey (revised) consists of 18 items that measure the frequency of sexual risk behaviors in the past 6 months (4 items were deleted from the original scale because of low reliability).An exploratory factor analysis on the original test revealed five factors, Sexual Risk Taking with Uncommitted Partners, Risky Sex Acts, Impulsive Sexual Acts, Intent to Engage in Risky Sexual Behaviors, and Risky Anal Sex Acts (3 items). For the five subscales, the Cronbach's alphas were $.89, .80, .77, .78$, and .57, respectively. The two-week test-retest reliabilities for the 5 subscales were $.90, .89, .81, .75$, and .58 , respectively. Risky anal sex acts was not included in the revised survey [17].

\section{Sexual Activity}

The frequency of general sexual activity was assessed by adding the responses to two separate questions that were attached to the Sexual Risk Survey: How many partners have you had sex with? How many partners have you engaged in sexual behavior with but not had intercourse with?

\section{Big Five Inventory-10}

The moderating variable, personality type, is measured using the short version of the Big Five Inventory (BFI-10), which determines one's level of openness, conscientiousness, extroversion, agreeableness, and neuroticism. Good test-retest reliability was established for a period of 8 weeks, showing significant correlations between total $(r=.72)$ and personality scale scores $(.64$ $<r<.80$ ) on the BFI-10 at time 1 and time 2 [18].

\section{Results}

Table I includes the descriptive statistical data for all the sexual behavior variables in each of the four sleep 


\section{Psychology \& Psychological Research International Journal}

groups. A multivariate analysis of covariance (MANCOVA) was conducted to explore the role of sleep health in sexual risk taking. The independent variable was sleep group (healthy sleepers, unhealthy sleepers, drowsy sleepers, and qualitatively impaired sleepers). The dependent variables were total sexual risk taking, sexual risk taking with uncommitted partners, risky sex acts, impulsive sexual behavior, and intent to engage in risky sexual behavior. Gender and personality were entered as covariates to control for individual differences. Sexual activity was also entered as a covariate to differentiate sexual risk taking from general sexual behavior (which is not necessarily risky).

The overall MANCOVA was significant, indicating that there is a significant effect of sleep health on sexual risk taking after controlling for sex, personality, and sexual activity, $\mathrm{F}(18,249.39)=6.15, \mathrm{p}<.000$, Wilks' $\Lambda=.35$, partial $\eta 2=.29$. Significant differences were found between the four sleep groups on measures of total sexual risk taking, $\mathrm{F}(3)=18.92, \mathrm{p}<.000$, risky sex acts, $\mathrm{F}(3)=$ $20.22, \mathrm{p}<.000$, impulsive sexual behavior, $\mathrm{F}(3)=6.66, \mathrm{p}<$ .000 , and intent to engage in risky sexual behavior, $F(3)=$ $4.73, \mathrm{p}<.000$. Sidak post-hoc pairwise comparisons were conducted to identify which sleep groups were significantly different. Unhealthy sleepers reported significantly more sexual risk taking $(\mathrm{M}=140.46, \mathrm{SD}=$ 4.604) than healthy sleepers $(M=66.75, S D=11.21$, $p<$ $.000)$, drowsy sleepers $(M=108.21, S D=10.28, p<.05)$, and qualitatively impaired sleepers $(\mathrm{M}=107.35, \mathrm{SD}=$ 5.15, p< .000). Drowsy and qualitatively impaired sleepers also reported significantly more sexual risk taking than healthy sleepers. There were no significant differences found between the sleep groups for sexual risk taking with uncommitted partners, $F(3)=2.37, p>$ .05. Unhealthy sleepers reported significantly more risky sex acts $(M=45.97, S D=2.87)$ than healthy $(M=6.88, S D$ $=7.00, \mathrm{p}<.000)$, drowsy $(\mathrm{M}=26.32, \mathrm{SD}=6.42, \mathrm{p}<.05)$, and qualitatively impaired sleepers $(\mathrm{M}=18.23, \mathrm{SD}=3.21$, $\mathrm{p}<.000)$. Unhealthy sleepers $(\mathrm{M}=18.26, \mathrm{SD}=1.30, \mathrm{p}<$ $.002)$, drowsy sleepers $(\mathrm{M}=17.53, \mathrm{SD}=2.91, \mathrm{p}<.05)$, and qualitatively impaired sleepers $(\mathrm{M}=22.64, \mathrm{SD}=1.46, \mathrm{p}<$ .000) all report significantly more impulsive sexual behaviors than healthy sleepers $(\mathrm{M}=5.75, \mathrm{SD}=3.17)$. Unhealthy sleepers reported significantly greater intent to engage in risky sexual behavior $(\mathrm{M}=35.79, \mathrm{SD}=1.93)$ than qualitatively impaired sleepers $(\mathrm{M}=26.98, \mathrm{SD}=$ $2.16, \mathrm{p}<.05)$.

In males, there is a significant effect of sleep health on sexual risk taking after controlling for sexual activity and personality, $\mathrm{F}(18,74.02)=25.43, \mathrm{p}<.000 ;$ Wilks' $\Lambda=.00$, partial $\eta 2=.84$. Significant differences in sexual risk taking with uncommitted partners, $F(3)=3.87, \mathrm{p}<.05$, risky sex acts, $\mathrm{F}(3)=5.44, \mathrm{p}<.01$, and intent to engage in risky sexual behavior, $F(3)=6.62, p<.01$ were found among the four sleep groups. Sidak post-hoc pairwise comparisons were conducted to identify significant differences between sleep groups. In males, unhealthy sleepers reported significantly more risky sex acts $(\mathrm{M}=$ $26.49, \mathrm{SD}=2.24)$ compared to healthy $(\mathrm{M}=11.40, \mathrm{SD}=$ $5.03, \mathrm{p}<.05)$, drowsy $(\mathrm{M}=7.76, \mathrm{SD}=4.44, \mathrm{p}<.01)$, and qualitatively impaired sleepers $(\mathrm{M}=15.44, \mathrm{SD}=2.23, \mathrm{p}<$ $.01)$. Unhealthy sleepers reported significantly less intent to engage in sexual risk taking $(\mathrm{M}=18.47, \mathrm{SD}=3.35)$ than drowsy $(\mathrm{M}=48.31, \mathrm{SD}=6.64, \mathrm{p}<.01)$ and qualitatively impaired sleepers $(M=43.40, S D=3.33, p<.001)$.

In females, there is a significant effect of sleep health on sexual risk taking after controlling for sexual activity and personality, $\mathrm{F}(18,136.250)=19.06, \mathrm{p}<.000$; Wilks' $\Lambda=$ .028 , partial $\eta 2=.70$. Significant differences in total sexual risk taking, $F(3)=80.96, p<.000$, sexual risk taking with uncommitted partners, $\mathrm{F}(3)=3.51, \mathrm{p}<.05$, risky sex acts, $\mathrm{F}(3)=19.28, \mathrm{p}<.000$, impulsive sexual behavior, $\mathrm{F}(3)=$ $30.00, \mathrm{p}<.000$, and intent to engage in risky sexual behavior, $F(3)=59.94, p<.000$ were found among the four sleep groups. Sidak post-hoc pairwise comparisons were conducted to identify significant differences between sleep groups. In females, unhealthy sleepers reported significantly greater sexual risk taking $(\mathrm{M}=$ 154.85, $\mathrm{SD}=3.04)$ than healthy $(\mathrm{M}=64.47, \mathrm{SD}=7.05, \mathrm{p}<$ $.000)$, drowsy, $(\mathrm{M}=121.51, \mathrm{SD}=6.90, \mathrm{p}<.000)$, and qualitatively impaired sleepers $(\mathrm{M}=104.86, \mathrm{SD}=3.51, \mathrm{p}<$ .000). Qualitatively impaired sleepers reported significantly more sexual risk taking with uncommitted partners $(\mathrm{M}=38.60, \mathrm{SD}=.91)$ than unhealthy sleepers $(\mathrm{M}$ $=34.60, \mathrm{SD}=.79, \mathrm{p}<.05)$. Unhealthy sleepers reported significantly more risky sex acts $(\mathrm{M}=59.19, \mathrm{SD}=4.16)$ than healthy $(\mathrm{M}=6.75, \mathrm{SD}=9.64, \mathrm{p}<.000)$ and qualitatively impaired sleepers $(M=20.733, S D=4.799$, $\mathrm{p}<.000)$. Unhealthy $(\mathrm{M}=19.70, \mathrm{SD}=.935, \mathrm{p}<.000)$, drowsy $(\mathrm{M}=16.60, \mathrm{SD}=2.12, \mathrm{p}<.000)$, and qualitatively impaired sleepers $(\mathrm{M}=23.54, \mathrm{SD}=1.08, \mathrm{p}<.000)$ reported significantly more impulsive sexual behavior than healthy sleepers $(\mathrm{M}=-1.10, \mathrm{SD}=2.17)$. Qualitatively impaired sleepers also report more impulsive sexual behavior than drowsy sleepers. Unhealthy sleepers reported significantly greater intent to engage in sexual risk taking $(\mathrm{M}=41.36, \mathrm{SD}=1.03)$ than healthy $(\mathrm{M}=24.80$, $\mathrm{SD}=2.39<.000)$, drowsy $(\mathrm{M}=28.55, \mathrm{SD}=2.34, \mathrm{p}<.000)$, and qualitatively impaired sleepers $(\mathrm{M}=21.99, \mathrm{SD}=1.19$, $\mathrm{p}<.000$ ). 


\section{Psychology \& Psychological Research International Journal}

\section{Hierarchical Multiple Regression}

Hierarchical multiple regressions were conducted to predict sexual risk taking. Separate analyses were done on the female sample and male sample. In males, sexual activity was a significant predictor, $\mathrm{R} 2=.54, \mathrm{~F}(1,39)=$ $45.97, \mathrm{p}<.000$, and accounted for $54.1 \%$ of the variance in sexual risk taking. Personality variables significantly improved the regression model, $\mathrm{R} 2=.71, \mathrm{~F}(6,34)=13.63$, $\mathrm{p}<.000$, and explained an additional $16.5 \%$ of the variance in sexual risk taking. Sleep health did not add to the regression model, only explaining $3.1 \%$ of the variance in sexual risk, $\mathrm{R} 2=.74, \mathrm{~F}(9,31)=9.68, \mathrm{p}<.000$. The full regression model, $\mathrm{R} 2=.76, \mathrm{~F}(11,29)=8.54, \mathrm{p}<$ .000 , was significant, and explained $76.4 \%$ of the variance in sexual risk taking. After all variables were entered into the equation, sexual activity $(\mathrm{t}=4.25, \mathrm{p}<.000, \eta 2=.15)$, unhealthy sleep $(\mathrm{t}=2.03, \mathrm{p}<.05, \eta 2=.03)$, and impaired sleep $(t=1.98, p<.05, \eta 2=.03)$ were the only significant predictors of sexual risk taking.

In females, sexual activity accounted for $73.3 \%$ of the variance in sexual risk taking, $\mathrm{R} 2=.73, \mathrm{~F}(1,61)=167.57$, $\mathrm{p}<.000$. Personality did not improve the regression model, accounting for a mere $1.7 \%$ of variance in sexual risk taking $R 2=.75, F(6,56)=28.07, \mathrm{p}<.000$. Sleep health was significant, $R 2=.96, F(9,53)=125.87, \mathrm{p}<.000$, and explained $20.5 \%$ of the variance in sexual risk.. The full model for sexual risk taking was significant, $\mathrm{R} 2=.96, \mathrm{~F}(11$, $51)=100.44, \mathrm{p}<.000$, with sexual activity $(\mathrm{t}=21.33, \mathrm{p}<$ $.000, \eta 2=.394)$, unhealthy sleep $(\mathrm{t}=11.78, \mathrm{p}<.000, \eta 2=$ $.12)$, drowsy sleep $(\mathrm{t}=5.67, \mathrm{p}<.000, \eta 2=.03)$, and impaired sleep $(\mathrm{t}=4.50, \mathrm{p}<.000, \eta 2=.02)$ being the only significant predictors.

\section{Discussion}

The purpose of the current study was to explore the role of sleep health in sexual risk taking. Additionally, this study makes an important distinction between sexual activity and sexual risk taking. While sexual risk taking is measured by specific risk behaviors (e.g. unprotected sex, multiple sexual partners), sexual activity is determined by an individual's overall sexual encounters. Looking at risk taking and sexual activity, independently, allowed us to compare sexual risk taking across individuals, independent of levels of sexual activity.

The results indicate that for both men and women, sleep affects the probability of engaging in risky sex but the effects appear to be much stronger for women. After controlling for the effect of sexual activity and personality in males, Unhealthy Sleepers reported more risky sex acts than Healthy, Drowsy, and Qualitatively impaired sleepers. Risky sex acts included sexual intercourse without a condom, sexual intercourse without birth control, oral sex without a condom, and sex under the influences of drugs or alcohol. Drowsy and Qualitatively Impaired Sleepers reported greater intent to engage in sexual risk taking than Unhealthy Sleepers. It is unclear why Unhealthy Sleepers report significantly less intent to engage in sexual risk taking. Perhaps the multiple sleep issues among Unhealthy Male Sleepers affects their level of personal insight or makes their plans for sexual encounters more idealistic and less realistic. Clearly further replication is needed before over interpreting this unexpected finding.

In females, total sexual risk taking, along with all four subscales (Sexual Risk Taking with Uncommitted Partners, Risky Sex Acts, Impulsive Sexual Behavior, and Intent to Engage in Sexual Risk Taking) showed significant variations according to sleep health. Unhealthy Female Sleepers reported more total sexual risk taking than Healthy, Drowsy, and Qualitatively Impaired Sleepers, after controlling for sexual activity and personality. Drowsy and Qualitatively Impaired Female Sleepers also reported significantly more total sexual risk taking than Healthy Sleepers. Like the males, Unhealthy Female Sleepers reported more risky sex acts than Healthy and Qualitatively Impaired sleepers. Unhealthy, Drowsy, and Qualitatively Impaired Female Sleepers reported significantly more impulsive sexual behavior than Healthy Sleepers. The effect is much stronger for the females.

Why would unhealthy sleep impact the sexual risk taking of women more consistently than men? Women tend to need more hours of sleep than men for optimal functioning and so Unhealthy Female Sleepers may make poorer decisions or be more vulnerable to sexual pressures than Unhealthy Male Sleepers. It could also be that men, are more dominant in sexual encounters and more likely than women to take (or not take) responsibility for safe sexual practices, independent of sleep health. However, the amount of variance in sexual risk taking accounted for by unhealthy sleep in so much greater in women than in men $(20.5 \%$ vs. $3.1 \%)$, compelling cultural explanations are in need of testing.

While males with either high daytime sleepiness or poor sleep quality reported a greater intent to engage in sexual risk taking, there was no actual change in the reported frequency of sexual risk taking. However, women with either daytime sleepiness or poor sleep quality reported significantly greater intent to engage in 


\section{Psychology \& Psychological Research International Journal}

sexual risk taking than Healthy Female Sleepers and also reported significantly more risky sexual behavior than Healthy Female Sleepers. This might be because contrary to popular belief, women have more of a say than men in whether or not risky sex proceeds during a sexual encounter or it could be because women know in advance whether they will submit to their own or their partner's desire for risky sex. Or perhaps there is a third unknown factor, influencing both risky intent and risky behavior in women that is not operating in men. For example, when sleep deprived, women have been shown to make less risky decisions then men [19]. So perhaps the decision to have risky sex may be less impulsive in women and more reflective of intent ("e.g. "He told me he was tested last month", "I just can tell he is not a high-risk partner", etc). Of course, if the risky behavior was done with intent, that opens whole new areas of concern. While popular thinking is that men contribute more to risky sex encounters, the current results suggest that, to the extent that intent is important, the situation may be more complicated.

This study also demonstrated the usefulness of creating categorical groups of sleepers so one can examine the cumulative effect of having both daytime sleepiness as well as poor sleep quality. It appears that for both men and women the two factors interact in such a way that the impact on decision making and impulsiveness, which has been independently documented, is often compounded in some exponential manner when they occur together. It suggests that it may be possible to identify a group of high risk Unhealthy Sleepers who could most benefit from either behavioral sleep medicine and/or sexual responsibility interventions.

Overall, these results suggest that sleep has important implications for one's sexual health, as unhealthy sleep is associated with more sexual risk taking. The translational implications of these findings are important for clinicians who wish to protect the health of their clients. Young adults, in particular, need to understand how sleep deprivation and poor sleep quality can compromise their sexual activity decisions. Consciousness raising about the link between poor sleep and risky sex is the first step in increasing the likelihood of safe sex practices. Creating action plans to guard against risky sex are more likely to be adopted and instituted once an individual has accepted that they have an increased likelihood of acting in an impulsive and self-defeating manner that they will regret.

Future studies examining cultural factors affecting sexual risk taking should include measures of sleep health and explore further gender differences in risk intent.

\begin{tabular}{|c|c|c|c|c|c|c|}
\hline & \multicolumn{4}{|c|}{ Sex } & \multirow{2}{*}{\multicolumn{2}{|c|}{ Total }} \\
\hline & \multicolumn{2}{|c|}{ Males } & \multicolumn{2}{|c|}{ Females } & & \\
\hline & $M$ & $S D$ & $M$ & $S D$ & $M$ & $S D$ \\
\hline Sexual Risk Taking & & & & & & \\
\hline Healthy Sleepers & 126.00 & 25.46 & 168.50 & 38.45 & 160.00 & 39.28 \\
\hline Unhealthy Sleepers & 130.47 & 55.57 & 160.56 & 43.86 & 147.57 & 50.93 \\
\hline Drowsy Sleepers & 91.33 & 28.43 & 120.60 & 94.10 & 109.63 & 74.30 \\
\hline Impaired Sleepers & 94.18 & 58.48 & 66.04 & 39.44 & 77.43 & 49.40 \\
\hline Total & 112.34 & 56.15 & 120.89 & 65.00 & 117.52 & 61.53 \\
\hline Uncommitted Partner & & & & & & \\
\hline Healthy Sleepers & 50.00 & 14.14 & 75.00 & 15.38 & 70.00 & 17.81 \\
\hline Unhealthy Sleepers & 56.95 & 35.00 & 36.76 & 16.22 & 45.48 & 27.60 \\
\hline Drowsy Sleepers & 22.67 & 8.08 & 36.20 & 38.00 & 31.13 & 29.88 \\
\hline Impaired Sleepers & 27.88 & 17.99 & 23.20 & 15.32 & 25.10 & 16.40 \\
\hline Total & 42.05 & 30.18 & 36.19 & 23.98 & 38.50 & 26.61 \\
\hline Risky Sex Acts & & & & & & \\
\hline Healthy Sleepers & 22.50 & 3.54 & 31.50 & 7.69 & 29.70 & 7.86 \\
\hline Unhealthy Sleepers & 28.00 & 12.86 & 60.24 & 28.98 & 46.32 & 28.27 \\
\hline Drowsy Sleepers & 8.67 & 4.93 & 38.00 & 40.96 & 27.00 & 34.59 \\
\hline Impaired Sleepers & 12.29 & 8.08 & 12.32 & 7.82 & 12.31 & 7.83 \\
\hline Total & 19.80 & 12.95 & 35.81 & 30.49 & 29.50 & 26.20 \\
\hline Impulsive Sex & & & & & & \\
\hline Healthy Sleepers & 26.00 & 1.41 & 19.88 & 9.61 & 21.10 & 8.88 \\
\hline
\end{tabular}




\section{Psychology \& Psychological Research International Journal}

\begin{tabular}{|c|c|c|c|c|c|c|}
\hline Unhealthy Sleepers & 17.47 & 9.91 & 21.08 & 10.25 & 19.52 & 10.15 \\
\hline Drowsy Sleepers & 19.67 & 6.43 & 17.00 & 13.02 & 18.00 & 10.52 \\
\hline Impaired Sleepers & 20.82 & 13.96 & 15.36 & 9.60 & 17.57 & 11.72 \\
\hline Total & 19.44 & 11.36 & 18.33 & 10.24 & 18.77 & 10.65 \\
\hline Intent & & & & & & \\
\hline Healthy Sleepers & 27.50 & 6.36 & 42.13 & 5.77 & 39.20 & 8.27 \\
\hline Unhealthy Sleepers & 28.05 & 6.17 & 42.48 & 8.11 & 36.25 & 10.24 \\
\hline Drowsy Sleepers & 40.33 & 20.43 & 29.40 & 17.29 & 33.50 & 17.94 \\
\hline Impaired Sleepers & 33.18 & 22.51 & 15.16 & 8.38 & 22.45 & 17.86 \\
\hline Total & 31.05 & 15.97 & 30.56 & 15.68 & 30.75 & 15.72 \\
\hline
\end{tabular}

Table 1: Descriptive Statistics for Sexual Behavior Variables by Sleep Group.

\section{References}

1. Shrier LA, Koren S, Aneja P, De Moor C (2010) Affect regulation, social context, and sexual intercourse in adolescents. Archives of Sexual Behavior 39(3): 695705.

2. Mahowald M, Schenck C (2005) Insights from studying human sleep disorders. Nature 437(7063): 1279-1285.

3. Minkel J, Banks S, Htaik O, Jones C, Mc Glinchey E (2012) Sleep deprivation and stressors: evidence for elevated negative affect in response to mild stressors when sleep deprived. Emotion 12(5): 1015-1020.

4. Lim J, Dinges D (2010) A meta-analysis of the impact of short-term sleep deprivation on cognitive variables. Psychol Bull 136(3): 375-389.

5. Christian M, Ellis A (2011) Mining the effects of sleep deprivation on workplace deviance: A self regulatory perspective. Academy of Management Journal 54 (5): 913-934.

6. Roberts RE, Roberts CR, Duong HT (2008) Chronic insomnia and its negative consequence for health and functioning of adolescents: A 12-month prospective study. J Adolesc Health 42: 294-302.

7. Rose S, Berg-Cross L, Crowell, Nancy A (2017) Sleep and Psychological abuse among cohabitating couples: A pilot study. Partner Abuse 8(4): 347-360.

8. Kilgore W, Balkin T, Wesensten N (2010) Impaired decision making following 49 hours of sleep deprivation. J Sleep Res 15(1): 7-13.

9. Cooper ML (2010) Toward a person X situation model of sexual risk-taking behaviors: Illuminating the conditional effects of traits across sexual situations and relationship contexts. J Pers Soc Psychol 98(2): 319-341.

10. Cooper ML, Shapiro CM, Powers AM (1998) Motivations for sex and risky sexual behavior among adolescents and young adults: A functional perspective. Journal of Personality and Social Psychology 75(6): 1528-1558.

11. Fortenberry JD, Temkit MH, Tu W, Graham CA, Katz BP, et al. (2005) Daily mood, partner support, sexual interest, and sexual activity among adolescent women. Health Psychol 24(3): 252-257.

12. MacApagal KR, Janssen E, Fridberg DJ, Finn PR, Heiman JR (2011) The effect of impulsivity, sexual arousability, and abstract intellectual ability on men's and women's Go/No-Go task performance. Arch Sex Behav 40(5): 995-1006.

13. Backhaus J, Junghanns K, Hohagen F (2004) Sleep disturbances are correlated with decreased morning awakening salivary cortisol. Psychoneuroendocrinology 29(9): 1184-1191.

14. Krystal A, Edinger J (2008) Measuring sleep quality. Sleep Med 9(S1): 10-17.

15. Buysse DJ, Hall ML, Strollo PJ, Kamarck TW, Owens J, et al. (2008) Relationships between the Pittsburgh Sleep Quality Index (PSQI), Epworth Sleepiness Scale (ESS), and Clinical Polysomnographic measures in a community sample. J Clin Sleep Med 4(6): 563-571.

16. Kendzerska T, Smith P, Brignardello-Peterson R, Leung R, Tomlinson G (2014) Evaluation of the measurement properties of the Epworth Sleepiness Scale: A Systemic Review. Sleep Med Rev 18(4): 321331. 


\section{Psychology \& Psychological Research International Journal}

17. Turchik J, Walsh K, Marcus D (2015) Confirmatory validation of the factor structure and reliability of the Sexual Risk Survey in a large multiuniversity sample of US students. International Journal of Sexual Health 27(2): 93-105.

18. Rammstedt B, John OP (2007) Measuring personality in one minute or less: A 10 item short version of the Big Five Inventory in English and German. Journal of Research in Personality 41(1): 203-212.
19. Ferrara M, Bottasso A, Tempesta D, Carrieri M, DeGennaro L, et al. (2015) Gender Differences in sleep deprivation effects on risk and inequality aversion: Evidence from an Economic experiment. PLoS One 10(3): e0120029.

20. Taylor M (2012) Sleep Deprivation, Attachment Patterns and Hooking Up. Unpublished master's thesis. Howard University.

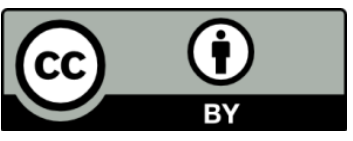

